\title{
AVALIAÇÃO DA TAXA DE DISPENSAÇÃO DE ISONIAZIDA UTILIZADA NO TRATAMENTO PREVENTIVO DE TUBERCULOSE EM SERVIÇO ESPECIALIZADO EM HIV/AIDS
}

\section{ARTIGO ORIGINAL}

SANTANA, Claudinei Alves ${ }^{[1]}$, GUTIERREZ, Eliana Battaggia [2]

SANTANA, Claudinei Alves. GUTIERREZ, Eliana Battaggia. Avaliação Da Taxa De Dispensação De Isoniazida Utilizada No Tratamento Preventivo De Tuberculose Em Serviço Especializado Em HIV/Aids. Revista Científica Multidisciplinar Núcleo do Conhecimento. Ano 06, Ed. 04, Vol. 03, pp. 05-21. Abril. ISSN: 2448-0959, Link de acesso: https://www.nucleodoconhecimento.com.br/saude/isoniazida-utilizada, DOI: 10.32749/nucleodoconhecimento.com.br/saude/isoniazida-utilizada

\section{RESUMO}

Introdução: A síndrome da imunodeficiência adquirida, causada pelo vírus da imunodeficiência humana constituiu-se em uma das principais epidemias de doenças infecciosas do século XX. A Tuberculose é uma doença crônica, infecciosa e contagiosa, causada pelo Mycobacterium tuberculosis. Ambas as doenças, interagem tornando-se um grande problema de saúde pública. $O$ tratamento preventivo com isoniazida apresenta opção para não manifestação da tuberculose. A adesão ao tratamento é essencial para sucesso da prevenção. Objetivo: Avaliar a taxa de adesão ao tratamento preventivo com isoniazida, medida através da dispensação do medicamento na farmácia e os fatores associados entre sujeitos com HIV/aids e Infecção Latente pelo Mycobacterium tuberculousis acompanhados em Serviço Especializado no Atendimento de Pacientes HIV/aids. Metodologia: Estudo retrospectivo e prospectivo, descritivo e analítico com pacientes adultos com HIV/aids em seguimento, Serviço de Extensão ao Atendimento de Pacientes HIV/aids da Divisão de Moléstias Infecciosas e Parasitárias do Hospital das Clínicas da Faculdade de Medicina da Universidade de São Paulo, em uso do Sistema de Informação e 
Gestão Hospitalar (SIGH) implantado nos Ambulatórios e Serviço de Farmácia, sendo realizando a análise de associação entre as variáveis de interesse e desfecho através do teste t de Student com IC de 95\%, sendo considerado com significado estatístico $p \leq 5 \%$. Resultados: Foram incluídos 161 sujeitos que participaram do estudo sendo $78,9 \%$ do sexo masculino, $67,7 \%$ idade inferior 50 anos, $87,0 \%$ com mais de 5 anos de infeção pelo HIV, e intercorrências durante o tratamento preventivo estiveram presentes em 12,4\% (20). A taxa de adesão ao tratamento preventivo com isoniazida em prontuário e prescrição eletrônica foram $96 \%$ e $83 \%$, respectivamente, ambas sendo superiores a $80 \%$ segundo recomendação da Organização Mundial de Saúde e não houve associação entre as variáveis de interesse e desfecho. Conclusão: A taxa elevada pode ser justificada parcialmente, por práticas adotadas na assistência médica e farmacêutica como o agendamento das datas de dispensação de medicamentos, unificação para dispensações de diferentes medicamentos na mesma data, informações na farmácia sobre fatores que elevam a efetividade do tratamento. A assistência e atenção farmacêutica realizada de forma adequada foi em última análise a grande responsável pelos resultados alcançados.

Palavras-chave: adesão ao TPI, prescrição eletrônica, serviço de farmácia, tuberculose, HIV/aids.

\section{INTRODUÇÃO}

A síndrome da imunodeficiência adquirida (aids), causada pelo vírus da imunodeficiência humana (HIV) constituiu-se em uma das principais epidemias de doenças infecciosas do século XX (BIGGAR, 1988; DE COOK et al, 2011). No final de 2019 estimava-se que cerca de 38 milhões de pessoas viviam com HIV em todo o mundo. (UNAIDS, 2020). No Brasil, de 1980 a junho de 2020, foram notificados 1.011.617 casos de aids no país. (BRASIL, 2020).

A Tuberculose (TB) é uma doença crônica, infecciosa e contagiosa, causada pelo Mycobacterium tuberculosis (Mtb) (VERONESI, 2005). Em 2018, cerca de 10 milhões de pessoas desenvolveram tuberculose, aproximadamente $9 \%$ estavam 
vivendo com o HIV. (UNAIDS, 2020). A TB é a segunda causa de morte por doença infecciosa no mundo com 1,5 milhões de óbitos em 2018. (UNAIDS, 2020).

No Brasil, em 2019, foram diagnosticados 73.864 casos novos de TB, o que correspondeu a um coeficiente de incidência de 35,0 casos/100 mil habitantes. (BRASIL, 2020).

A infecção por HIV é um dos principais fatores de risco para o desenvolvimento da TB (EL-SADR et al, 2008; STERLING et al, 2010). A comorbidade TB e HIV traz problemas especiais para o diagnóstico da TB, para a terapêutica de ambas as doenças e consome uma grande parcela dos recursos de saúde em países em desenvolvimento (FÄTKENHEUER et al, 1999). A TB está entre as principais causas de morte entre os indivíduos com aids nestas regiões (BLANC et al, 2007).

$O$ tratamento de pacientes coinfectados requer 0 uso de medicamentos tuberculostáticos e antirretrovirais usados concomitantemente, e traz como desafios aos pacientes o alto número de comprimidos, adesão, possibilidade de interações medicamentosas com sobreposição de efeitos tóxicos e síndrome de reconstituição imune inflamatória (PADMAPRIYADARSINI et al, 2011).

Umas das medidas para a prevenção da TB, em indivíduos infectados pelo Mtb e com risco aumentado de evoluir para doença, é o tratamento preventivo com isoniazida (TPI) da Infecção Latente por Mtb (ILMtb) (CONDE et al, 2009; LEUNG et al, 2010; HORSBURGH; RUBIN, 2011; DUARTE et al, 2007). A ILMTb é a presença de Mtb numa fase não ativa, sem produzir sintomas clínicos. Estima-se que até um terço da população mundial esteja infectado de forma latente pelo Mtb (AKOLO et al, 2010). $O$ TPI pode reduzir o risco em pessoas vivendo com HIV/aids (PVHA) de 33 a $67 \%$ por até 48 meses. A Organização Mundial de Saúde e o Ministério da Saúde, a fim de reduzir o risco de TB em PVHA recomendam administração de isoniazida (INH), na dose de 5 a $10 \mathrm{mg} / \mathrm{kg} /$ dia de peso até $300 \mathrm{mg}$ por 6 meses (540 comprimidos) consecutivos para o tratamento de PVHA com Teste Tuberculínico $\geq 5$ milímetros, desde que a TB ativa, atual ou anterior, tenha sido excluída (CONDE et al, 2009). Importante salientar o risco de elevação assintomática de enzimas hepáticas como a AST (aspartato aminotransferase), ALT (alanina aminotransferase) e GGT (gamaglutamiltransferase) após o início do tratamento com INH. (CHURCHYARD et al, 2007; COHN, 2000). 
O conceito de adesão varia entre os autores. Pode ser definido como sendo o quanto o paciente segue as orientações de um agente de saúde, com as quais concordou, e considera-se como aderente ao TPI o paciente que ingere pelo menos $80 \%$ das doses prescritas (WHO, 2003).

A avaliação da adesão à terapia com medicamentos pode ser feita de forma direta e indireta, com métodos bioquímicos, objetivos, subjetivos e clínicos, que são os mais utilizados mundialmente (GORENOI et al, 2007).

Um dos métodos de a avaliação da adesão à terapia pode ser através da dispensação de medicamento na farmácia, e para tanto é necessária a disponibilidade de sistemas que registrem, com precisão, a dispensação aos pacientes (OIGMAN, 2003).

O Serviço de Farmácia é parte integrante da assistência nos Serviços de Assistência Especializados em HIV/aids segundo a Organização Mundial de Saúde (WHO, 2008). Sendo também de grande importância a atuação do farmacêutico clínico em infectologia e equipe de farmácia na assistência às PVHA (VAN MIL et al, 2006).

\section{OBJETIVO}

Este trabalho teve como objetivo primário avaliar a taxa de adesão ao TPI, medida através da quantidade de comprimidos dispensados de isoniazida, correlacionado com a quantidade prescrita em prontuário e quantidade prescrita na prescrição eletrônica e os fatores associados entre sujeitos com HIV/aids e ILMtb em Serviço Especializado no Atendimento de Pacientes HIV/aids.

\section{METODOLOGIA}

\section{DESENHO DE ESTUDO}

Estudo retrospectivo e prospectivo, descritivo e analítico com pacientes adultos com HIV/aids em seguimento em Serviço de Extensão ao Atendimento de Pacientes HIV/aids da Divisão de Moléstias Infecciosas e Parasitárias do Hospital das Clínicas da Faculdade de Medicina da Universidade de São Paulo. (SEAP HIV/Aids), um 
ambulatório especializado em HIV/aids, com Sistema de Informação e Gestão Hospitalar (SIGH) implantado nos Ambulatórios para elaboração de prescrição eletrônica e no Serviço de Farmácia para dispensação de medicamentos. A pesquisa foi aprovada pela Comissão de Ética para Análise de Projetos de Pesquisa da Faculdade de Medicina da Universidade de São Paulo sob o número 1068/08.

\section{SELEÇÃO DOS SUJEITOS}

Foram incluídos sujeitos que apresentaram pelo menos 1 prescrição de $\mathrm{INH}$ no Serviço de Farmácia do SEAP HIV/Aids no período de 01/07/2008 a 30/06/2011. Foram excluídos sujeitos com TB anterior e no momento do estudo, que não realizaram acompanhamento no período do estudo e que iniciaram o TPI antes de 01/07/2008.

\section{COLETA DE DADOS}

Foram obtidos dados em prontuário por meio de um formulário padronizado, para todo o período de acompanhamento do sujeito no serviço; do SIGH foram obtidos dados através de relatórios de gestão gerados nos computadores localizados no Serviço de Farmácia.

A taxa de dispensação de INH no Serviço de Farmácia, desfecho deste estudo, de INH foi calculada como a relação percentual entre o número de comprimidos dispensados pela farmácia e a estimativa do número comprimidos prescritos, conforme o registro em prontuário das datas de início e de fim do TPI relatada no prontuário e a quantidade de comprimidos prescritos de forma eletrônica (QCP), foi calculada através do intervalo em dias entre a data da $1^{\circ}$ prescrição de $\mathrm{INH}$ registrada no SIGH e a data de encerramento multiplicado pelo número de comprimidos/dia. $(300 \mathrm{mg}=3 \mathrm{cp})$. 


\section{ANÁLISE ESTATÍSTICA}

A análise estatística foi realizada com o programa SPSS - Statistics, versão 17.0. Foi realizada análise descritiva das variáveis com medidas de tendência central (média, mediana, desvio padrão, mínima e máxima) e proporções, e o teste t de Student para as associações, com IC de $95 \%$, sendo considerado com significado estatístico $p \leq$ $5 \%$.

\section{RESULTADOS}

\section{CARACTERÍSTICAS DA AMOSTRA}

Foram selecionados inicialmente 203 sujeitos que apresentaram prescrição de INH no período de 01/07/2008 a 30/06/2011, sendo que, após aplicar os critérios de exclusão, foram incluídos 161 sujeitos (figura 1) 
Figura 1 - Algoritmo de inclusão de sujeitos

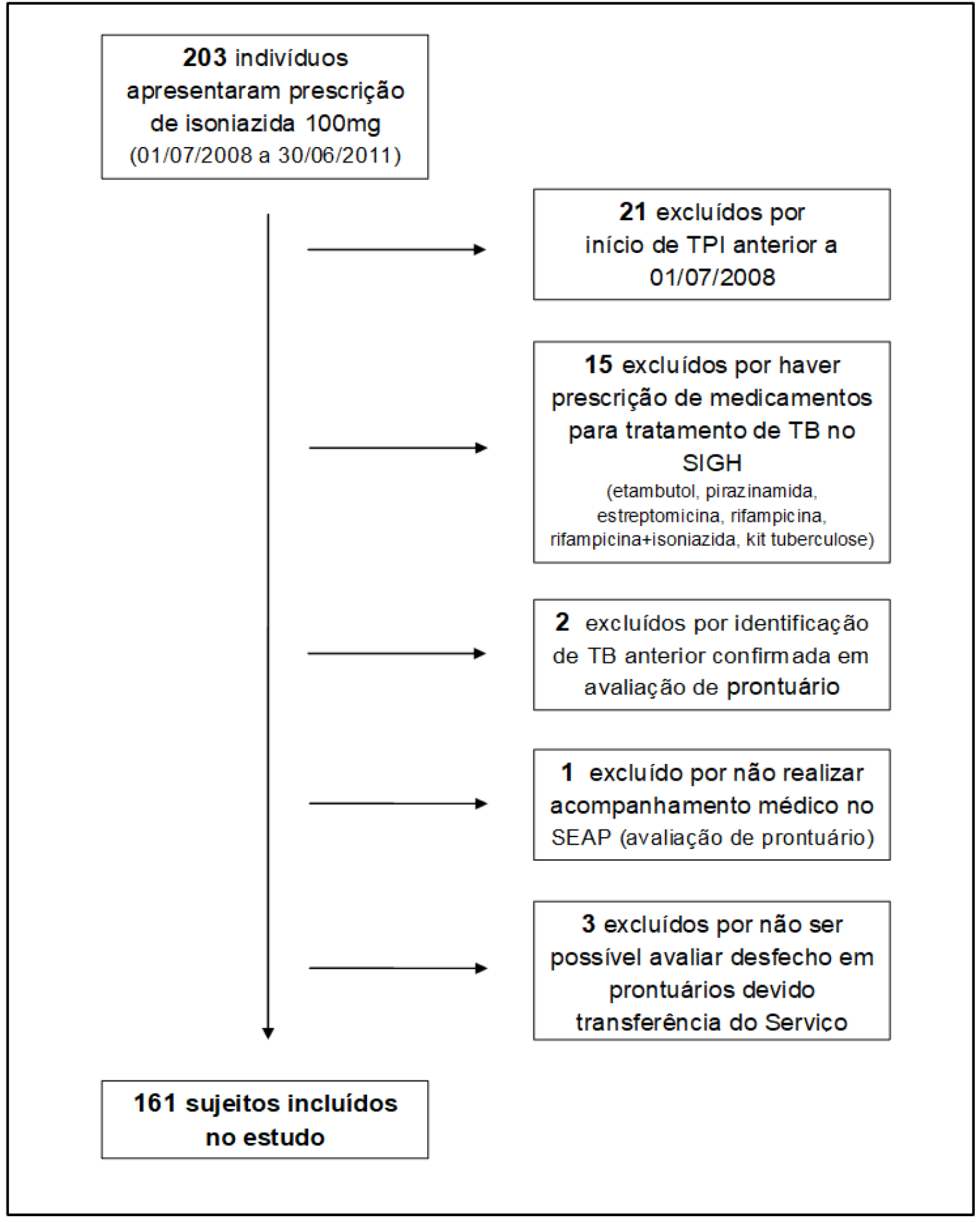

Fonte: Elaborado pelo autor 
Tabela 1- Características dos sujeitos. SEAP HIV/Aids, HC-FMUSP, São Paulo, 2008 a 2011

\begin{tabular}{|c|c|c|}
\hline Variável & $\mathbf{N}$ & $\%$ \\
\hline \multicolumn{3}{|l|}{ Sexo } \\
\hline$M$ & 127 & 78,9 \\
\hline $\mathrm{F}$ & 34 & 21,1 \\
\hline \multicolumn{3}{|l|}{ Idade } \\
\hline$<50$ & 109 & 67,7 \\
\hline$\geq 50$ & 52 & 32,3 \\
\hline \multicolumn{3}{|c|}{ Doença Concomitante } \\
\hline Sim & 125 & 77,6 \\
\hline Não & 36 & 22,4 \\
\hline \multicolumn{3}{|c|}{ Uso de drogas lícitas ou ilicitas } \\
\hline Sim & 36 & 22,4 \\
\hline Não & 125 & 77,6 \\
\hline \multicolumn{3}{|l|}{ Anos Inf. HIV } \\
\hline$\leq 4$ anos & 21 & 13,0 \\
\hline$\geq 5$ anos & 140 & 87,0 \\
\hline \multicolumn{3}{|l|}{ Uso de TARV } \\
\hline Sim & 149 & 92,5 \\
\hline Não & 12 & 7,5 \\
\hline \multicolumn{3}{|c|}{ Núm. de comp. ARV } \\
\hline$\leq 3 \mathrm{cp}$ & 52 & 34,9 \\
\hline$\geq 4 \mathrm{cp}$ & 97 & 65,1 \\
\hline \multicolumn{3}{|c|}{ Acompanhamento Médico } \\
\hline assistente & 136 & 84,5 \\
\hline residente & 25 & 15,5 \\
\hline
\end{tabular}

Fonte: Elaborado pelo autor 
Figura 2- Algoritmo de intercorrências durante o TPI

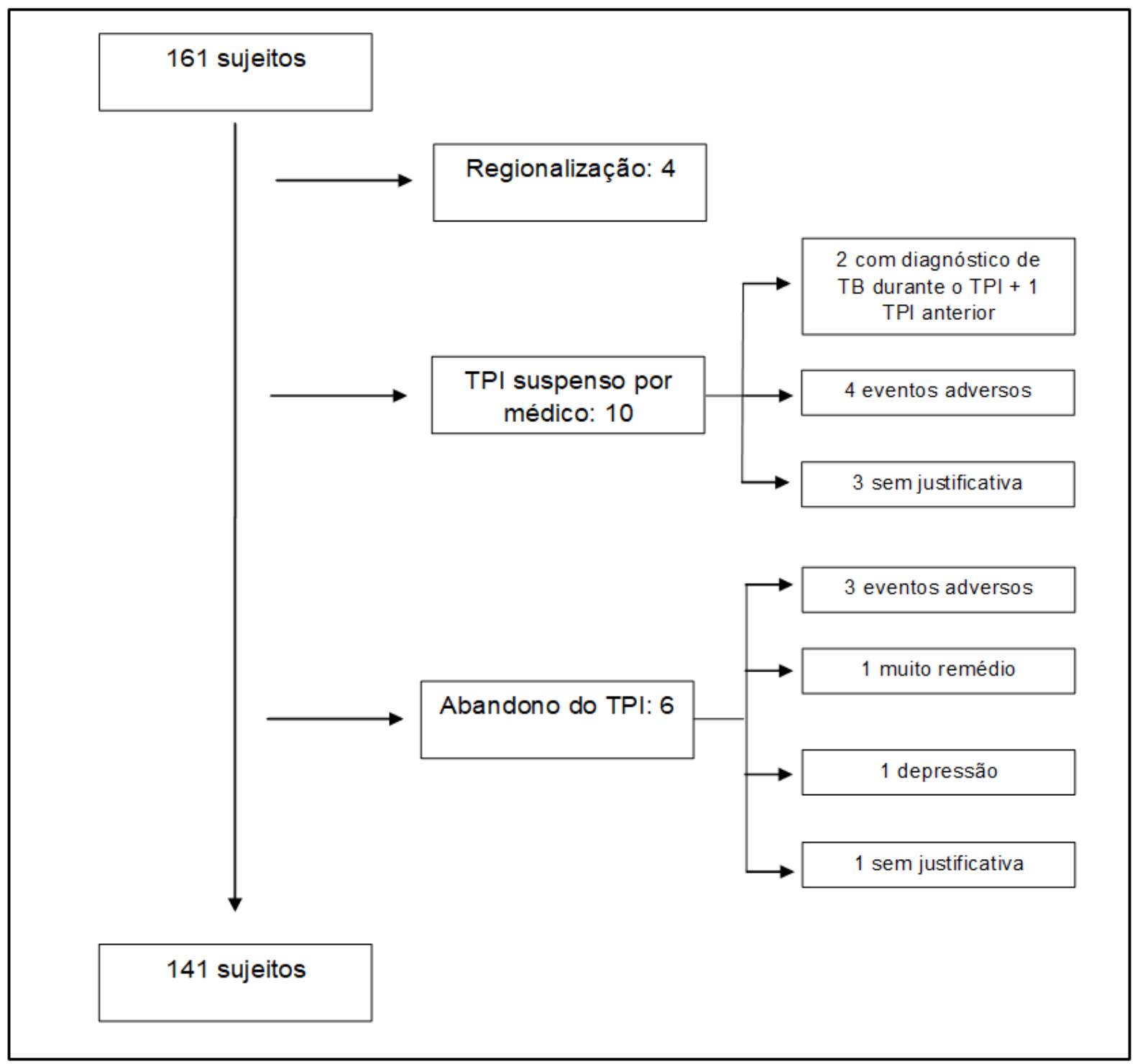

Fonte: Elaborado pelo autor

\section{AVALIAÇÃO DE ADESÃO DESCRITA EM PRONTUÁRIO}

A avaliação de adesão ao TPI foi registrada em prontuário para 13\% (21) dos sujeitos, sendo considerada boa em $24 \%$ (5) dos mesmos. Na avaliação de adesão em sujeitos que utilizavam TARV havia informação em prontuário para 53\% (79) sujeitos, sendo considerada boa em $41 \%$ (32). 
Gráfico 1. Avaliação da adesão ao TPI descrito em prontuário. SEAP HIV/Aids, HC-FMUSP, São Paulo -2008 a 2011

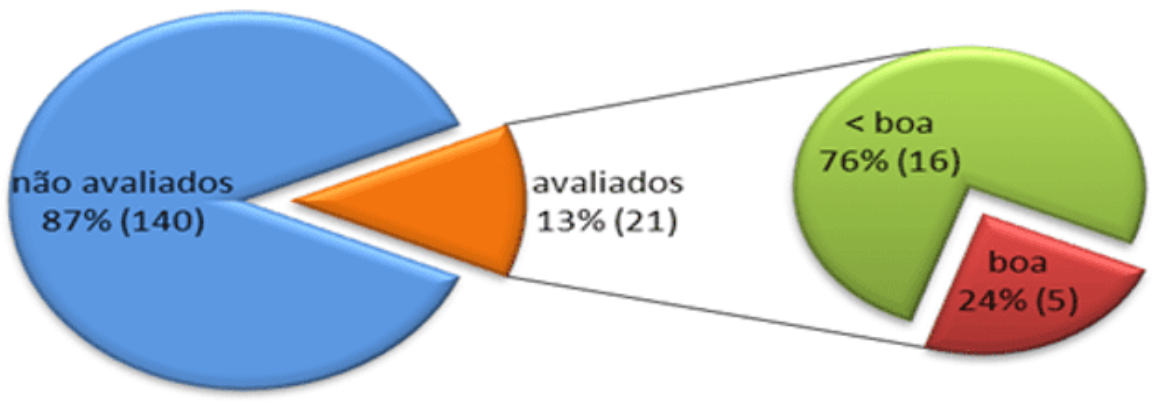

Fonte: Elaborado pelo autor

Tabela 3- Distribuição de sujeitos de acordo com resultado de AST, ALT e GGT para avaliação de hepatotoxicidade nos períodos pré, concomitante e pós TPI. SEAP HIV/Aids, HC-FMUSP, São Paulo 2008 a 2011

\begin{tabular}{|c|c|c|c|c|c|c|}
\hline \multirow[t]{3}{*}{ Exames } & \multirow[t]{3}{*}{ Período } & \multicolumn{4}{|c|}{ Sujeitos } & \multirow{2}{*}{$\frac{\text { Total }}{*}$} \\
\hline & & \multicolumn{2}{|c|}{$<37 \mathrm{U} / \mathrm{L}$} & \multicolumn{2}{|c|}{$\geq 37 \mathrm{U} / \mathrm{L}$} & \\
\hline & & $\mathrm{N}$ & $\%$ & $\mathrm{~N}$ & $\%$ & \\
\hline & Pre TPI & 142 & 88,2 & 19 & 11,8 & 161 \\
\hline \multirow[t]{5}{*}{ AST } & Concom itante ao TPI & 107 & 71,3 & 43 & 26,7 & 150 \\
\hline & Pós TPI & 130 & 86,1 & 21 & 13,9 & 151 \\
\hline & & \multicolumn{2}{|c|}{$<41 \mathrm{U} / \mathrm{L}$} & \multicolumn{2}{|c|}{$\geq 41 \mathrm{U} / \mathrm{L}$} & ** \\
\hline & & $\mathrm{N}$ & $\%$ & $\mathrm{~N}$ & $\%$ & \\
\hline & Pre TPI & 136 & 85,0 & 24 & 15,0 & 160 \\
\hline \multirow[t]{5}{*}{ ALT } & Concom itante ao TPI & 107 & 71,3 & 43 & 28,7 & 150 \\
\hline & Pós TPI & 118 & 77,6 & 34 & 22,4 & 152 \\
\hline & & \multicolumn{2}{|c|}{$\leq 61 \mathrm{U} / \mathrm{L}$} & \multicolumn{2}{|c|}{$\geq 62 \mathrm{U} / \mathrm{L}$} & 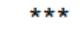 \\
\hline & & $\mathrm{N}$ & $\%$ & $\mathrm{~N}$ & $\%$ & \\
\hline & Pre TPI & 112 & 75,2 & 37 & 24,8 & 149 \\
\hline \multirow[t]{2}{*}{ GGT } & Concom itante ao TPI & 99 & 76,7 & 30 & 23,3 & 129 \\
\hline & Pós TPI & 95 & 74,8 & 32 & 25,2 & 127 \\
\hline
\end{tabular}

Fonte: Elaborado pelo autor

RC: 81915

Disponível em: https://www. nucleodoconhecimento.com.br/saude/isoniazida-utilizada 
Tabela 4- Comprimidos de INH prescritos, de acordo com prontuário e prescrição, e a quantidade de comprimidos dispensados pela farmácia, em média e mediana, por sujeito, durante o TPI. SEAP HIV/Aids, HC-FMUSP, São Paulo - 2008 a 2011

\begin{tabular}{llllll}
\hline \multicolumn{2}{c}{ Variável } & \multicolumn{4}{c}{ Número de Comprimidos de Isoniazida } \\
\cline { 2 - 6 } & Média & Mediana & DP & Min - Max \\
\hline \multicolumn{2}{c}{ Cp. Prescritos } & & & & \\
& Prontuário & 573,2 & 550 & 162,94 & $12-1386$ \\
& Prescrição & 685,8 & 639 & 267,84 & $84-1837$ \\
Cp. Disp. & Farmácia & 504,9 & 530 & 150,18 & $90-850$ \\
\hline
\end{tabular}

Cp: comprimido; Disp: dispensado

146 sujeitos inclui dos

Fonte: Elaborado pelo autor

Tabela 5- Mediana da Adesão ao TPI, de acordo com a taxa de dispensação de INH, calculada com base em informações do prontuário e conforme a prescrição eletrônica. SEAP HIV/Aids, HC-FMUSP, São Paulo - 2008 a 2011

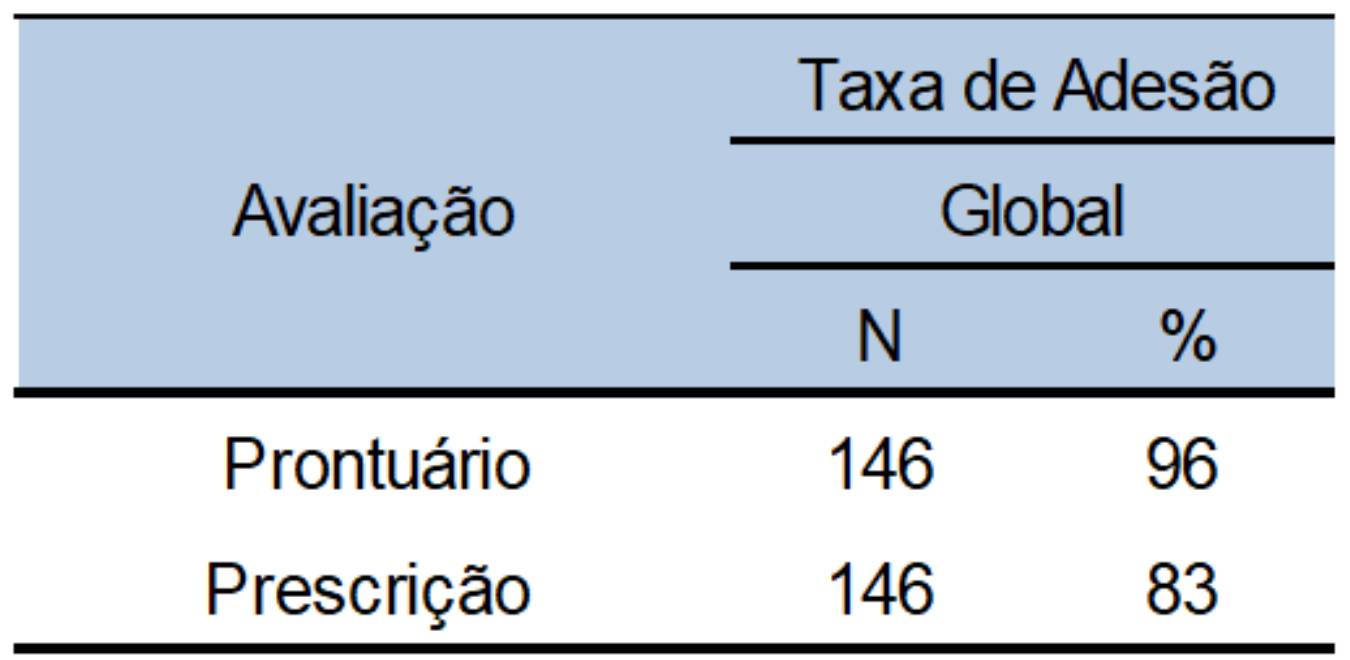

Fonte: Elaborado pelo autor 
Tabela 6- Taxa de adesão à INH de sujeitos HIV/aids de acordo com variáveis de interesse, SEAP HIV/Aids, HC-FMUSP, São Paulo - 2008-2011

\begin{tabular}{|c|c|c|c|}
\hline Variáveis & $\mathbf{N}$ & \% ade são & $\mathbf{p}$ \\
\hline \multicolumn{4}{|l|}{ Sexo } \\
\hline M & 114 & 89,7 & 0,589 \\
\hline $\mathrm{F}$ & 31 & 92,2 & \\
\hline \multicolumn{4}{|l|}{ Idade } \\
\hline$<50$ & 98 & 88,8 & 0,261 \\
\hline$\geq 50$ & 47 & 93,3 & \\
\hline \multicolumn{4}{|l|}{ Anos Inf. HIV } \\
\hline$\leq 4$ anos & 20 & 84,9 & 0,247 \\
\hline$\geq 5$ anos & 125 & 91,1 & \\
\hline \multicolumn{4}{|c|}{ Doença Concomitante } \\
\hline Sim & 112 & 91,6 & 0,174 \\
\hline Não & 33 & 85,6 & \\
\hline \multicolumn{4}{|c|}{ Uso de Drogas Lícitas/lícitas } \\
\hline Sim & 30 & 83,6 & 0,066 \\
\hline Não & 115 & 91,9 & \\
\hline \multicolumn{4}{|l|}{ Uso de TARV } \\
\hline Sim & 134 & 91,2 & 0,305 \\
\hline Não & 11 & 79,3 & \\
\hline \multicolumn{4}{|l|}{ Núm. de cp ARV/dia } \\
\hline$\leq 3 \mathrm{cp}$ & 45 & 89,6 & 0,536 \\
\hline$\geq 4 \mathrm{cp}$ & 89 & 91,9 & \\
\hline \multicolumn{4}{|c|}{ Acompanhamento Médico } \\
\hline assistente & 123 & 90,7 & 0,602 \\
\hline residente & 22 & 88,0 & \\
\hline \multicolumn{4}{|c|}{ 1. Avaliação médica do TPI } \\
\hline$\leq 30$ dias & 22 & 95,2 & 0,296 \\
\hline$\geq 31$ dias & 121 & 89,9 & \\
\hline \multicolumn{4}{|c|}{ CV pré TPI em TARV* } \\
\hline indetectável & 115 & 90,7 & 0,560 \\
\hline detectável & 19 & 93,7 & \\
\hline \multicolumn{4}{|c|}{ CV conc. TPI em TARV** } \\
\hline indetectável & 118 & 91,4 & 0,768 \\
\hline detectável & 8 & 93,6 & \\
\hline $\begin{array}{l}\text { NOTA: Teste } t \text {-student } \\
\text { cp: co mprimidos: } \mathrm{CV} \text { : carg a viral: } \\
\text { inf: inf ecção; núm: número:TARV } \\
\text { sujeit os sem result ad os lab o r at or }\end{array}$ & vírus & $\begin{array}{l}\text { Inod eficiência hum } \\
\text { to preventivo com }\end{array}$ & niazida \\
\hline
\end{tabular}

Fonte: Elaborado pelo autor 


\section{DISCUSSÃO E CONCLUSÂO}

Em nosso estudo não identificamos fatores associados à adesão ao TPI. A literatura especializada é controversa em relação e este aspecto: enquanto, em alguns estudos, de forma semelhante ao nosso, idade, sexo, tempo de infecção, doenças concomitantes, drogas ilícitas, TARV, acompanhamento médico, condição virológica e adesão ao TPI (TULSKY et al, 2000; SHUKLA et al, 2002), não tiveram associação com a adesão ao TPI outros mostraram associação com raça/cor, idade, moradia, álcool, evento adverso e país de origem (LOBUE et al, 2003; TULSKY et al, 2000; PARSYAN et al, 2007).

Dos 161 sujeitos, 12,4\% (20) sofreram intercorrências durante o TPI, estes pacientes foram excluídos das análises da adesão, pois, tais intercorrências influenciaram negativamente a continuidade dos pacientes ao tratamento. No entanto nessas intercorrências não foram relatados eventos como hepatotoxicidade.

Em relação ao risco de hepatotoxicidade (elevação dos marcadores de função hepática) avaliado através dos resultados de exames laboratoriais, 12,4\% dos pacientes apresentaram algum tipo de intercorrência, a hepatotoxicidade, efeito adverso da INH bem definido e documentado (CONDE et al, 2009; CHURCHYARD et al, 2007; COHN; O'BRIEN, 2000; CONH, 2000), ocorreu de forma discreta e transitória, e não acarretou suspensão do TPI. Aparentemente, a preocupação com adesão não se refere a todos os tratamentos de forma homogênea e neste trabalho identificamos que enquanto $53 \%$ dos sujeitos tinham registro em prontuário da avaliação de adesão ao TARV apenas 13\% tinham avaliação relativa ao TPI. Por ser tratar de um tratamento para reduzir o risco de TB e potencialmente hepatóxico seria desejável maior preocupação com sua adesão (CONDE et al, 2009; COHN et al, 2000; COHN, 2000).

Há diferentes metodologias para avaliação de adesão ao TPI, variando entre proporção de frascos fornecidos comparado ao previsto, número de pacientes que finalizaram tratamento, proporção de comprimidos dispensados que foram retornados à farmácia, banco de dados com informações sobre a dispensação de medicamentos e autorrelato. (HIRANSUTHIKUL et al, 2005; WHO, 2008; TULSKY et al, 2000; SHUKLA et al, 2002; KABALI et al, 2011; BATKI et al, 2002; WHITE et al, 2003). 
Neste trabalho a taxa de adesão ao TPI através das informações de prontuário e informações de prescrição eletrônica foram de 96 \% e 83\% respectivamente, ambas sendo superior a $80 \%$, que inicialmente consideramos adequado para que o TPI seja eficiente (VEENING, 1968; FALLAB-STUBI et al, 1998; HIRANSUTHIKUL et al, 2005) A elevada taxa de adesão ao TPI observada pode ser atribuída a características do serviço, reduzido tempo de espera, horário de funcionamento estendido, crenças pessoais, a própria doença, entre outros (GUSMAO et al, 2006; WHO, 2003, REINERS et al, 2008; NEVES et al, 2010). Esta taxa pode ser justificada, pelo menos parcialmente, por práticas adotadas na assistência, médica e farmacêutica: entre 2006, a 2008 o serviço de farmácia do SEAP HIV/Aids passou a agendar as datas de dispensação de medicamentos, unificar as dispensações de diferentes medicamentos na mesma data, informar os médicos, através de notificação em prontuário, sobre não comparecimento do paciente para retirada de medicamentos do TARV e de INH e, principalmente, esclarecer os pacientes, individualmente, sobre fatores que elevam a efetividade do tratamento, destacando-se a importância da ingestão do medicamento conforme as orientações recebidas e sua dispensação na data agendada. A assistência farmacêutica realizada de forma adequada, tendo por horizonte a elevação da qualidade de saúde e de vida dos pacientes, foi, em última análise, a grande responsável pelo resultado alcançado.

\section{CONSIDERAÇÔES FINAIS}

A adesão aos tratamentos recomendados é essencial para que se alcance o resultado esperado. Adesão é um comportamento complexo, assim como sua avaliação, nesse estudo há algumas limitações como a ausência de outro método de avaliação de adesão, como entrevista ao profissional médico ou paciente que poderiam colaborar para identificação de dificuldades nas questões relacionadas a adesão por ambos os indivíduos. No entanto, mesmo com as limitações foi possível identificar a adesão ao TPI com participação ativa das informações disponibilizadas pelo Serviço de Farmácia. 


\section{REFERÊNCIAS}

AKOLO, Christopher et al. Treatment of latent tuberculosis infection in HIV infected persons. Cochrane database of systematic reviews, n. 1, 2010.

BATKI, Steven L. et al. A controlled trial of methadone treatment combined with directly observed isoniazid for tuberculosis prevention in injection drug users. Drug and alcohol dependence, v. 66, n. 3, p. 283-293, 2002.

BIGGAR, R. J. AIDS: a global problem. Cancer detection and prevention, v. 12, n. 1-6, p. 169-174, 1988.

BLANC, François-Xavier et al. Treatment strategies for HIV-infected patients with tuberculosis: ongoing and planned clinical trials. The Journal of infectious diseases, v. 196, n. Supplement_1, p. S46-S51, 2007.

BRASIL. Ministério da Saúde. Secretaria de Vigilância em Saúde. Boletim Epidemiológico Tuberculose. Brasília (DF): Ministério da Saúde; 2020. Disponível em: $<\quad$ http://www.aids.gov.br/pt-br/pub/2020/boletim-epidemiologico-de-turbeculose2020>. Acesso em 28 de fev. de 2021.

BRASIL. Ministério da Saúde. Secretaria de Vigilância em Saúde. Departamento de DST, Aids e Hepatite Virais. Boletim Epidemilógico Especial- Aids 2020. Brasília (DF): Ministério da Saúde; 2020. Disponível em: < http://www.aids.gov.br/ptbr/pub/2020/boletim-epidemiologico-hivaids-2020>. Acesso em 28 de fev. de 2021.

CHURCHYARD, Gavin J. et al. Tuberculosis preventive therapy in the era of HIV infection: overview and research priorities. The Journal of infectious diseases, $v$. 196, n. Supplement_1, p. S52-S62, 2007.

COHN, David L. et al. Targeted tuberculin testing and treatment of latent tuberculosis infection. MMWR Morb Mortal Wkly Rep, v. 49, n. 6, p. 1-54, 2000.

COHN, David L. Treatment of latent tuberculosis infection: renewed opportunity for tuberculosis control. Clinical infectious diseases, v. 31, n. 1, p. 120-124, 2000. 
CONDE, Marcus Barreto et al. III Diretrizes para tuberculose da Sociedade Brasileira de Pneumologia e Tisiologia. Jornal Brasileiro de Pneumologia, v. 35, n. 10, p. 1018-1048, 2009.

DE COCK, Kevin M.; JAFFE, Harold W.; CURRAN, James W. Reflections on 30 years of AIDS. Emerging infectious diseases, v. 17, n. 6, p. 1044, 2011.

DE GUSMÃO, Josiane Lima; MION JR, Décio. Adesão ao tratamento-conceitos. Rev Bras Hipertens vol, v. 13, n. 1, p. 23-25, 2006.

DUARTE, Raquel et al. Tratamento da tuberculose latente: Revisão das normas, 2006. Revista Portuguesa de Pneumologia (English Edition), v. 13, n. 3, p. 397418, 2007.

EL-SADR, Wafaa Mahmoud; TSIOURIS, Simon. HIV-associated tuberculosis: diagnostic and treatment challenges. 2008.

ESTATISTICAS. UNAIDS. Disponível em: https://unaids.org.br/estatisticas/>. Acesso em 25 de fev. de 2021.

FALLAB-STUBI, C. L. et al. Electronic monitoring of adherence to treatment in the preventive chemotherapy of tuberculosis. The International Journal of Tuberculosis and Lung Disease, v. 2, n. 7, p. 525-530, 1998.

FÄTKENHEUER, Gerd et al. The return of tuberculosis. Diagnostic microbiology and infectious disease, v. 34, n. 2, p. 139-146, 1999.

GORENOI, Vitali; SCHÖNERMARK, Matthias P.; HAGEN, Anja. Interventions for enhancing medication compliance/adherence with benefits in treatment outcomes. GMS health technology assessment, v. 3, 2007.

HIRANSUTHIKUL, N. et al. INH preventive therapy among adult HIV-infected patients in Thailand. The International Journal of Tuberculosis and Lung Disease, v. 9, n. 3, p. 270-275, 2005. 
HORSBURGH JR, C. Robert; RUBIN, Eric J. Latent tuberculosis infection in the United States. New England Journal of Medicine, v. 364, n. 15, p. 1441-1448, 2011

KABALI, C. et al. Completion of isoniazid preventive therapy and survival in HIVinfected, TST-positive adults in Tanzania. The International journal of tuberculosis and lung disease, v. 15, n. 11, p. 1515-1522, 2011.

LEUNG, Chi Chiu et al. Treatment of latent infection with Mycobacterium tuberculosis: update 2010. European Respiratory Journal, v. 37, n. 3, p. 690-711, 2011.

LOBUE, Philip A.; MOSER, Kathleen S. Use of isoniazid for latent tuberculosis infection in a public health clinic. American journal of respiratory and critical care medicine, v. 168, n. 4, p. 443-447, 2003.

NEVES, Lis Aparecida de Souza; REIS, Renata Karina; GIR, Elucir. Adesão ao tratamento por indivíduos com a coinfecção HIV/tuberculose: revisão integrativa da literatura. Revista da Escola de Enfermagem da USP, v. 44, n. 4, p. 1135-1141, 2010.

OIGMAN, Wille. Métodos de avaliação da adesão ao tratamento antihipertensivo. Rev Bras Hipertens, v. 13, n. 1, p. 30-4, 2006.

PADMAPRIYADARSINI, C.; NARENDRAN, G.; SWAMINATHAN, Soumya. Diagnosis \& treatment of tuberculosis in HIV co-infected patients. The Indian journal of medical research, v. 134, n. 6, p. 850, 2011.

PARSYAN, Armen E. et al. Predictors of failure to complete treatment for latent tuberculosis infection. Journal of infection, v. 54, n. 3, p. 262-266, 2007.

REINERS, Annelita Almeida Oliveira et al. Produção bibliográfica sobre adesão/nãoadesão de pessoas ao tratamento de saúde. Ciência \& Saúde Coletiva, v. 13, p. 2299-2306, 2008.

SHUKLA, Sunita J. et al. Factors associated with the treatment of latent tuberculosis infection among health-care workers at a midwestern teaching hospital. Chest, v. 122, n. 5, p. 1609-1614, 2002. 
STERLING, Timothy R.; PHAM, Paul A.; CHAISSON, Richard E. HIV InfectionRelated tuberculosis: Clinical manifestations and treatment. Clinical Infectious Diseases, v. 50, n. Supplement_3, p. S223-S230, 2010.

TULSKY, Jacqueline Peterson et al. Adherence to isoniazid prophylaxis in the homeless: a randomized controlled trial. Archives of internal medicine, v. 160, n. 5, p. 697-702, 2000.

VAN MIL, JW Foppe; SCHULZ, Martin. A review of pharmaceutical care in community pharmacy in Europe. Harvard Health Policy Review, v. 7, n. 1, p. 155-168, 2006.

VEENING, G. J. Long term isoniazid prophylaxis. Controlled trial on INH prophylaxis after recent tuberculin conversion in young adults. Bulletin of the International Union against Tuberculosis, v. 41, p. 169-171, 1968.

VERONESI R. Tratado de Infectologia. 3aㅡ ed. São Paulo: Atheneu; 2005. p.1139206

WHITE M, C. et al. Effect of directly observed preventive therapy for latent tuberculosis infection in San Francisco. The International Journal of Tuberculosis and Lung Disease, v. 7, n. 1, p. 30-35, 2003.

WORLD HEALTH ORGANIZATION et al. Adherence to long-term therapies: evidence for action. World Health Organization, 2003.

WORLD HEALTH ORGANIZATION et al. Operations manual for delivery of HIV prevention, care and treatment at primary health centres in high-prevalence, resourceconstrained settings: Edition 1 for fieldtesting and country adaptation. 2008.

[1] Farmacêutico Clínico em Infectologia. Mestre em Ciências Médicas. Especialista em Oncologia Multiprofissional. Especialista em Planejamento Educacional e Docência do Ensino Superior. Especialista em Farmácia Hospitalar.

[2] Médica Infectologista. Doutora em Patologia. 
Enviado: Março, 2021.

Aprovado: Abril, 2021. 